\title{
Impaired integration of disambiguating evidence in delusional schizophrenia patients
}

\author{
N. Sanford ${ }^{1,2}$, R. Veckenstedt ${ }^{3}$, S. Moritz ${ }^{3}$, R. P. Balzan ${ }^{4}$ and T. S. Woodward ${ }^{1,2 *}$ \\ ${ }^{1}$ Department of Psychiatry, University of British Columbia, Vancouver, BC, Canada \\ ${ }^{2} B C$ Mental Health and Addiction Research Institute, Vancouver, BC, Canada \\ ${ }^{3}$ Department of Psychiatry and Psychotherapy, Clinical Neuropsychology, University Medical Centre Hamburg-Eppendorf, Germany \\ ${ }^{4}$ School of Psychology, Flinders University, Australia
}

Background. It has been previously demonstrated that a cognitive bias against disconfirmatory evidence (BADE) is associated with delusions. However, small samples of delusional patients, reliance on difference scores and choice of comparison groups may have hampered the reliability of these results. In the present study we aimed to improve on this methodology with a recent version of the BADE task, and compare larger groups of schizophrenia patients with/without delusions to obsessive-compulsive disorder (OCD) patients, a population with persistent and possibly bizarre beliefs without psychosis.

Method. A component analysis was used to identify cognitive operations underlying the BADE task, and how they differ across four groups of participants: (1) high-delusional schizophrenia, (2) low-delusional schizophrenia, (3) OCD patients and (4) non-psychiatric controls.

Results. As in past studies, two components emerged and were labelled 'evidence integration' (the degree to which disambiguating information has been integrated) and 'conservatism' (reduced willingness to provide high plausibility ratings when justified), and only evidence integration differed between severely delusional patients and the other groups, reflecting delusional subjects giving higher ratings for disconfirmed interpretations and lower ratings for confirmed interpretations.

Conclusions. These data support the finding that a reduced willingness to adjust beliefs when confronted with disconfirming evidence may be a cognitive underpinning of delusions specifically, rather than obsessive beliefs or other aspects of psychosis such as hallucinations, and illustrates a cognitive process that may underlie maintenance of delusions in the face of counter-evidence. This supports the possibility of the BADE operation being a useful target in cognitive-based therapies for delusions.

Received 14 June 2013; Revised 28 January 2014; Accepted 1 February 2014; First published online 27 February 2014

Key words: Bias against disconfirmatory evidence (BADE), delusions, obsessive-compulsive disorder (OCD), reasoning, schizophrenia.

\section{Introduction}

Delusions, one of the key symptoms of schizophrenia, are defined as fixed false beliefs that (i) are maintained despite counter-evidence and rational counterargument, (ii) would be dismissed by members of the same social and cultural environment, and (iii) are held with great conviction (APA, 2000). Maintenance of delusions despite counter-evidence and rational counter-argument involves explaining away disconfirming evidence by integrating it into the delusional framework, and this process has been described as

* Address for correspondence: T. S. Woodward, Ph.D., Room A3-A116, BC Mental Health and Addiction Research Institute, Translational Research Building, 3rd Floor, 938 W. 28th Avenue, Vancouver, BC, Canada V5Z 4H4.

(Email: todd.woodward@ubc.ca) safety behaviours (e.g. 'the only reason they did not shoot me yesterday was because I took another route to work') and incorporation (e.g. 'the only reason they did not shoot me yesterday was because they want me to suffer another day'; Garety et al. 2001; Freeman et al. 2002). There is also evidence that people with delusions have greater belief inflexibility relative to healthy controls (Colbert et al. 2010; So et al. 2010). Past work has shown that a cognitive bias against disconfirmatory evidence (BADE) exists in schizophrenia that is observable on delusion-neutral material, tapping into a cognitive process that may underlie the fixed aspect of delusions (i.e. maintained despite counter-evidence and rational counter-argument), possibly resulting in safety behaviours and incorporation. This bias is present in schizophrenia (Moritz \& Woodward, 2006a; Woodward et al. 2008) and is particularly enhanced in delusional patients (Moritz \& Woodward, 2006b; 


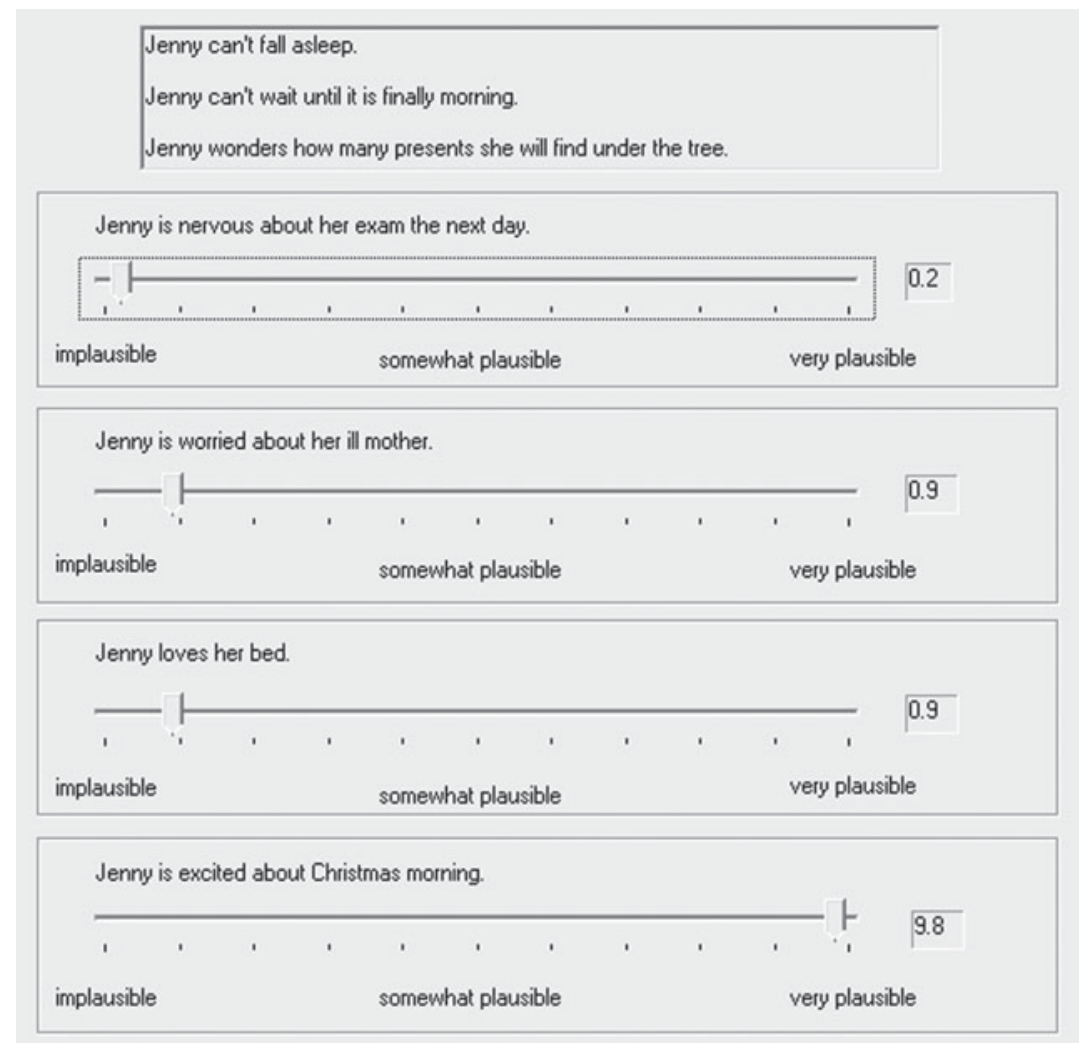

Fig. 1. A typical bias against disconfirmatory evidence (BADE) trial after all three statements have been presented.

Woodward et al. 2006; Riccaboni et al. 2012). Moreover, a previous factor analysis by Woodward et al. (2007) that incorporated BADE and traditional neuropsychological measures demonstrated that BADE measures were independent from other cognitive domains, including executive function as measured on the Wisconsin Card Sorting Test (WCST; Loong, 1991) and the Trail Making Test (TMT; Reitan \& Wolfson, 1985).

The most comprehensive form of the BADE task involves a set of scenarios that are accompanied by four possible interpretations, individually rated after each of three successively presented statements (illustrated in Fig. 1): one absurd interpretation, which seems implausible from the first statement and remains so throughout the trial; two lure interpretations (one neutral and one emotional), which seem plausible initially but are disconfirmed after the second or third statement; and one true interpretation, which does not seem to be the most plausible from the start but is confirmed by the final statement. For example, the statement 'Jenny can't fall asleep' may be interpreted in several ways (e.g. 'Jenny is worried about her exam the next day' or 'Jenny is excited about Christmas morning'); as more statements are presented (e.g. 'Jenny can't wait until it is finally morning'), it becomes increasingly clear as to which interpretations are lures, which are true, and which are absurd. The BADE has been characterized as an unwillingness to down-rate initially plausible interpretations ('lures') as they are revealed to be implausible (Moritz \& Woodward, 2006a,b; Woodward et al. 2006, 2008; Buchy et al. 2007).

Previous analyses of the BADE task have typically focused on lure interpretation ratings (Woodward et al. 2007) and/or have used rating adjustment scores as an index of BADE (Moritz \& Woodward, 2006b; Woodward et al. 2006, 2008; Veckenstedt et al. 2011). However, these methods do not take into account potential relationships between all BADE items. A more recent multivariate analysis of the BADE task (Speechley et al. 2012) that used all plausibility ratings in a factor analysis suggested that two cognitive operations underlie the BADE task, termed: (1) 'evidence integration', which showed that participants providing high ratings for 'lure' interpretations following the second and third statements rated low for 'true' interpretations on statement three, and vice versa, indexing the degree to which they integrated disambiguating evidence, and (2) 'response conservatism', dominated by ratings for lure interpretations after the first two sentences, in addition to all ratings for true interpretations; this component was labelled 'conservatism' because it seemed to arise from some participants being 
reluctant to provide high ratings when justified. Of note, severely delusional schizophrenia patients showed higher evidence integration scores than psychiatric and non-psychiatric controls, reflecting higher ratings for disconfirmed interpretations and lower ratings for confirmed interpretations.

Small sample sizes can be problematic in studies where a group of psychiatric subjects is divided into symptom-related subgroups. Although the analysis by Speechley et al. (2012) provided important insights into the cognitive operations underlying the BADE task, their study was limited by a small sample size for the delusional group $(n=10)$. One aim of the present study was to replicate this analysis in a larger group of schizophrenia patients that could be divided into adequately sized subgroups. In addition, the Speechley et al. (2012) study compared delusional and non-delusional schizophrenia patients with nondelusional bipolar patients to control for impairment in cognitive function, genetic and environmental susceptibility factors, and stigmatization associated with mental illness (Barrett et al. 2009). A potentially more interesting psychiatric control may be obsessivecompulsive disorder (OCD) patients, who generally have little symptom overlap with schizophrenia but do typically experience intrusive thoughts and obsessive beliefs from which they find it difficult to disengage. OCD is not a psychotic disorder, but there are similarities between delusions in psychosis and obsessions in OCD in the sense that, unlike the worries present in anxiety disorders (which are typically excessive concerns about realistic situations), obsessions in OCD are considered to be egodystonic and more bizarre in nature (Barlow, 2002). The inclusion of an OCD psychiatric control group should help to determine whether BADE is a cognitive bias specific to psychosis and delusions in particular, or can be observed in other individuals with obsessive and generally unrealistic and possibly bizarre beliefs.

In the current study, to negate concerns around sample size insufficiencies and other methodological idiosyncrasies in our past work as described above, we entered all interpretation ratings (absurd, neutral lure, emotional lure, and true) into a principal component analysis (PCA) to explore the cognitive underpinnings of the BADE paradigm and how they differ across patient groups and healthy individuals. Based on our previous analyses of schizotypy (Woodward et al. 2007) and schizophrenia data on small samples (Speechley et al. 2012), we anticipated that two factors would emerge from all BADE items, and that highly delusional schizophrenia patients would show the most impairment in evidence integration relative to healthy controls, OCD patients and schizophrenia patients with mild/no delusions.

\section{Method}

\section{Participants}

The present analysis was drawn from a consolidation of two datasets (total $n=214$ ): (1) previously published data (see Veckenstedt et al. 2011 for recruitment details) for 30 healthy controls with no psychiatric history, 54 patients fulfilling DSM-IV criteria for schizophrenia or schizo-affective disorder, and 20 OCD patients, and (2) unpublished data for 110 patients with schizophrenia or schizo-affective disorder. Thus, a total of 164 schizophrenia patients were included in the analysis. Schizophrenia patients were mainly recruited from an acute psychosis ward at the University Medical Centre, Department of Psychiatry and Psychotherapy in Hamburg, Germany. All diagnoses, including screening for the healthy control group, were determined by an experienced psychologist using the Mini International Neuropsychiatric Interview (MINI; Sheehan et al. 1998). None of the participants had previously completed a BADE task. All participants provided written informed consent after a complete description of the study, which was approved by the local ethics committee.

General exclusion criteria for all psychiatric participants were: (1) history of stroke, brain trauma, epilepsy, or other major neurological disorders, (2) severe substance dependence, and (3) an additional Axis I diagnosis (although an exception was made for major depression). An additional exclusion criterion for healthy individuals was the presence of any Axis I disorder, as determined using the MINI. Finally, OCD patients showing psychotic symptoms were excluded from participation. In the schizophrenia sample, current psychopathology was assessed with the Positive and Negative Syndrome Scale (PANSS; Kay et al. 1989). Patients with schizophrenia who reached a score of 4 or higher on delusions (item 1 on the positive subscale of the PANSS) were classified as high-delusional $(n=43)$.

\section{BADE task}

Full details of the German-language BADE task administration are described elsewhere (Veckenstedt et al. 2011). A screen shot of a typical BADE trial is presented in Fig. 1. In brief, the task consisted of 24 scenarios, eight of which were control trials (i.e. the 'true' interpretation was evident from the start of the trial rather than increasing in plausibility with accumulating evidence). Eight of the experimental trials and four control trials included only two possible interpretations (i.e. one lure and one true). All stimuli were presented on a computer screen. According to the plausibility evaluations of the participant, the 
Table 1. Sociodemographic characteristics of each participant group

\begin{tabular}{lllll}
\hline Variable & $\begin{array}{l}\text { Healthy } \\
(n=30)\end{array}$ & $\begin{array}{l}\text { OCD } \\
(n=20)\end{array}$ & $\begin{array}{l}\text { Low-delusional } \\
(n=121)\end{array}$ & $\begin{array}{l}\text { High-delusional } \\
(n=43)\end{array}$ \\
\hline Gender (M/F), $n$ & $19 / 11$ & $9 / 11$ & $72 / 49$ & $26 / 17$ \\
Age (years), mean (s.D.) & $32.23(8.28)$ & $30.30(9.55)$ & $33.89(10.80)$ & $\begin{array}{l}37.09(11.55) \\
\text { Education (years), mean (s.D.) }\end{array}$ \\
Verbal IQ, mean (s.D.) & $11.20(1.38)$ & $10.85(1.53)$ & $11.62(1.534)$ & $11.07(1.79)$ \\
& $108.31(10.63)$ & $104.95(11.93)$ & $108.87(14.45)^{*}$ & $102.55(11.75)$ \\
\hline
\end{tabular}

OCD, Obsessive-compulsive disorder; M, male; F, female; s.D., standard deviation.

${ }^{*} p<0.05$ for post-hoc comparison with high-delusional group.

instructor moved a slider along a scale of 0 ('implausible') to 10 ('very plausible') for each possible interpretation by using the computer mouse. The participants could use the entire range of scale in 0.1 steps to make their ratings. A small box to the right of each scale showed the current rating. Participants completed one practice trial at the start of the experiment.

\section{Ethical standards}

All procedures contributing to this work complied with the ethical standards of the relevant national and institutional committees on human experimentation and with the Declaration of Helsinki of 1975, as revised in 2008.

\section{Results}

\section{Sample characteristics}

Table 1 presents a list of group means for sample characteristics. The four groups did not differ in gender distribution $\left[\chi^{2}(3)=1.90, p>0.5\right]$. There were trendlevel group effects for age $\left(F_{3,210}=2.34, p=0.074\right)$, verbal intelligence, assessed by the Multiple Choice Vocabulary Test $\left(F_{3,208}=2.62, p=0.052\right)$, and years of education $\left(F_{3,208}=2.39, p=0.070\right)$. Post-hoc comparisons using the Tukey honestly significant difference (HSD) test showed a significant difference in verbal IQ between the delusional [mean $=102.55$, S.D. $=11.75$, 95\% confidence interval (CI) 98.89-106.21] and non-delusional groups (mean=108.87, S.D. $=14.45$, 95\% CI 106.27-111.47; $p=0.042)$, but otherwise no significant differences were found on age, IQ or education for any group comparisons (all $p^{\prime} \mathrm{s}>0.08$ ).

\section{PCA}

Control trials and trials that included only two possible interpretations were not included in the present analysis, resulting in 12 BADE variables: mean absurd, neutral lure, emotional lure and true interpretation ratings following each of the three evidence-providing statements (mean plausibility ratings for each group of participants are listed in Table 2). As in our previous study with a smaller sample size (Speechley et al. 2012), PCA was used for data reduction, with one difference: the PCA was run on the schizophrenia sample only, and component scores were subsequently constructed for the groups not included, using the coefficients obtained from this PCA. The reason for this was that, if group differences are present on the BADE and all participants are analysed simultaneously, this could lead to components that are specific to these group differences and would not emerge within any of the individual groups. Therefore, these ratings were entered into a PCA with varimax (orthogonal) rotation, in the schizophrenia group only (i.e. the main population of interest; $n=164$, no missing data). The overall Kaiser-MeyerOlkin (K-M-O) measure of sampling adequacy was 0.776 , with individual measures of sampling adequacy for each variable ranging from 0.693 to 0.897 . Bartlett's Test of Sphericity yielded $\chi^{2}(66)=1637.05(p<0.001)$, verifying that the correlation matrix was not an identity matrix. The determinant of the correlation matrix was greater than zero $\left(3.20 \times 10^{-5}\right)$, supporting an absence of multicollinearity.

An examination of the eigenvalues $(5.61,2.45,1.18$, $0.73,0.62,0.45,0.29,0.20,0.17,0.16,0.08,0.08)$ suggested a two-component solution, which accounted for $67.14 \%$ of the total variance. Components with eigenvalues greater than but close to 1.0 were not retained because the eigenvalues $>1.0$ rule is thought to lead to overextraction (Cliff, 1988; Jackson, 1993; Bandalos \& Boehm-Kaufman, 2008), and this was supported by a parallel analysis of eigenvalues obtained from analogous matrices of random values generated over 100 repetitions (see Hayton et al. 2004 for an in-depth description of this method). Component loadings are listed in Table 3, with component 1 labelled 'conservatism' and component 2 labelled 'evidence integration', in line with our previous factor analysis of the BADE task (Speechley et al. 2012). The rotation sums of squared loadings showed that the rotated 
Table 2. Mean (S.D.) plausibility ratings following each piece of evidence

\begin{tabular}{|c|c|c|c|c|c|}
\hline Statement & $\begin{array}{l}\text { Healthy } \\
(n=30)\end{array}$ & $\begin{array}{l}\text { OCD } \\
(n=20)\end{array}$ & $\begin{array}{l}\text { Low-delusional } \\
(n=121)\end{array}$ & $\begin{array}{l}\text { High-delusional } \\
(n=43)\end{array}$ & $\begin{array}{l}\text { All } \\
(n=214)\end{array}$ \\
\hline \multicolumn{6}{|l|}{ BADE trials } \\
\hline Absurd 1 & $0.94(0.81)$ & $0.92(0.92)$ & $1.13(1.15)$ & $1.67(1.50)$ & 1.19 (1.19) \\
\hline Absurd 2 & $0.43(0.55)$ & $0.56(0.58)$ & $0.65(0.81)$ & $1.30(1.59)$ & $0.74(1.01)$ \\
\hline Absurd 3 & $0.15(0.55)$ & $0.13(0.38)$ & $0.35(0.65)$ & $0.83(1.50)$ & $0.40(0.89)$ \\
\hline N lure 1 & $5.04(1.24)$ & $5.03(1.30)$ & 4.53 (1.97) & $4.54(1.70)$ & 4.65 (1.78) \\
\hline $\mathrm{N}$ lure 2 & $3.59(0.96)$ & $3.58(1.13)$ & $3.88(1.66)$ & 4.18 (1.59) & $3.87(1.52)$ \\
\hline $\mathrm{N}$ lure 3 & $0.97(1.00)$ & $0.66(0.98)$ & $1.50(1.55)$ & $2.04(1.62)$ & $1.46(1.50)$ \\
\hline E lure 1 & $4.50(1.55)$ & $4.75(1.40)$ & $4.34(1.94)$ & $4.45(1.84)$ & $4.42(1.82)$ \\
\hline E lure 2 & 3.49 (1.12) & 3.59 (1.23) & $3.82(1.74)$ & $4.16(1.72)$ & $3.82(1.62)$ \\
\hline E lure 3 & $1.09(0.93)$ & $0.84(1.18)$ & $1.32(1.39)$ & $1.85(1.75)$ & 1.35 (1.42) \\
\hline True 1 & 3.11 (1.09) & $3.05(1.31)$ & $2.83(1.64)$ & 3.04 (1.59) & $2.93(1.53)$ \\
\hline True 2 & $5.13(1.24)$ & $5.26(1.14)$ & $4.36(1.64)$ & $4.61(1.49)$ & $4.60(1.55)$ \\
\hline True 3 & $9.30(0.70)$ & $9.43(0.69)$ & $8.88(1.45)$ & $8.37(1.53)$ & 8.89 (1.36) \\
\hline \multicolumn{6}{|l|}{ Control trials } \\
\hline Absurd 1 & $0.54(0.74)$ & $0.46(0.61)$ & $0.73(1.10)$ & $1.28(1.44)$ & $0.79(1.12)$ \\
\hline Absurd 2 & $0.09(0.31)$ & $0.08(0.28)$ & $0.30(0.62)$ & $0.90(1.53)$ & $0.37(0.88)$ \\
\hline Absurd 3 & $0.03(0.18)$ & $0.06(0.28)$ & $0.21(0.47)$ & $0.92(1.74)$ & $0.32(0.91)$ \\
\hline N lure 1 & $1.98(1.18)$ & $2.06(1.31)$ & $1.87(1.55)$ & $2.29(1.74)$ & 1.99 (1.52) \\
\hline $\mathrm{N}$ lure 2 & $0.67(0.91)$ & $0.94(0.91)$ & $0.87(1.15)$ & $1.43(1.89)$ & $0.96(1.30)$ \\
\hline $\mathrm{N}$ lure 3 & $0.28(0.60)$ & $0.36(0.86)$ & $0.46(0.83)$ & $1.01(1.66)$ & $0.54(1.06)$ \\
\hline E lure 1 & $2.18(1.48)$ & $2.03(1.70)$ & $2.33(1.59)$ & $2.52(1.89)$ & $2.32(1.65)$ \\
\hline E lure 2 & $1.11(1.13)$ & $0.78(0.83)$ & $1.31(1.45)$ & 1.77 (1.73) & $1.33(1.44)$ \\
\hline E lure 3 & $0.43(0.95)$ & $0.17(0.51)$ & $0.77(1.21)$ & $1.21(1.59)$ & $0.75(1.25)$ \\
\hline True 1 & $6.32(1.48)$ & $6.95(1.82)$ & $5.84(2.10)$ & $5.91(2.19)$ & $6.03(2.03)$ \\
\hline True 2 & $8.41(1.25)$ & $8.43(1.58)$ & 7.75 (1.93) & $7.69(2.03)$ & 7.89 (1.85) \\
\hline True 3 & $9.54(0.57)$ & $9.32(0.97)$ & 8.88 (1.59) & $8.50(1.80)$ & $8.94(1.52)$ \\
\hline
\end{tabular}

OCD, Obsessive-compulsive disorder; BADE, bias against disconfirmatory evidence; N, neutral; E, emotional; S.D., standard deviation.

Control trials differed from BADE trials in that the 'true' interpretation was evident from the start of the trial rather than increasing in plausibility.

component 1 ('conservatism') accounted for $36.59 \%$ of the total variance and the rotated component 2 ('evidence integration') accounted for approximately $30.55 \%$ of the total variance.

The highest-loading variables on the response conservatism component were the first two ratings for neutral and emotional lure interpretations in addition to all ratings for true interpretations (see Table 3). Given the set-up of the BADE task (i.e. lure interpretations seem to be the most plausible initially and true interpretations seem somewhat less plausible initially but gradually become the most plausible), we would expect most participants to provide relatively high initial lure ratings and progressively higher true ratings. However, the existence of intercorrelations among these variables, reflected by the response conservatism component, suggests that a portion of the subjects generally rate low even though high ratings would be justified; that is, they are 'conservative' in their ratings (i.e. using caution or moderation). In comparison, evidence integration did not seem to be driven simply by an unwillingness to rate low when justified, as the negative loading of the third true rating suggests that this pattern arises from subjects continuing to rate high for initial beliefs following disconfirmatory evidence and continuing to rate low for the true interpretation following confirmatory evidence (see Table 3 ). This suggests that all BADE task ratings can generally be interpreted within the context of the two cognitive operations, response conservatism and evidence integration.

\section{Group by component analysis}

Component scores for the OCD and healthy control groups were computed by applying the component score coefficients obtained from running PCA on the schizophrenia group. The computed component scores for response conservatism and evidence integration 
Table 3. Varimax-rotated component loadings for BADE indicators (schizophrenia patients only, $n=164$ )

\begin{tabular}{llr}
\hline & \multicolumn{2}{l}{ Component } \\
\cline { 2 - 3 } Rating & Conservatism & $\begin{array}{l}\text { Evidence } \\
\text { integration }\end{array}$ \\
\hline Absurd 1 & 0.413 & $\mathbf{0 . 6 6 3}$ \\
Absurd 2 & 0.245 & $\mathbf{0 . 8 3 5}$ \\
Absurd 3 & 0.062 & $\mathbf{0 . 8 5 8}$ \\
N lure 1 & $\mathbf{0 . 8 9 3}$ & 0.042 \\
N lure 2 & $\mathbf{0 . 7 1 0}$ & $\mathbf{0 . 3 1 1}$ \\
N lure 3 & 0.189 & $\mathbf{0 . 7 4 7}$ \\
E lure 1 & $\mathbf{0 . 8 2 4}$ & 0.173 \\
E lure 2 & $\mathbf{0 . 7 5 6}$ & $\mathbf{0 . 3 5 4}$ \\
E lure 3 & 0.188 & $\mathbf{0 . 7 6 9}$ \\
True 1 & $\mathbf{0 . 7 9 9}$ & 0.331 \\
True 2 & $\mathbf{0 . 7 8 8}$ & 0.080 \\
True 3 & $\mathbf{0 . 5 2 5}$ & $-\mathbf{0 . 5 2 2}$ \\
\hline
\end{tabular}

N, Neutral; E, emotional.

All loadings corresponding to those $>0.40$ in Speechley et al. (2012) are set in bold font to show similarities in component patterns.

were entered as dependent variables into a one-way multivariate analysis of variance (MANOVA), with the four participant groups entered as a fixed factor, to determine whether conservatism and/or evidence integration were able to discriminate the highdelusional schizophrenia group $(n=43$; mean delusions $=4.51$, S.D. $=0.74)$ from the three control groups [(1) low-delusional schizophrenia, $n=121$; mean delusions $=1.59$, S.D. $=0.80$, (2) OCD, $n=20$, and (3) healthy controls, $n=30]$. This analysis revealed a significant main effect of group $\left(\Lambda_{\text {Wilks }}=0.875, F_{6,418}=4.821, p<\right.$ 0.001). Follow-up univariate ANOVAs revealed a significant between-groups effect for evidence integration $\left(F_{3,210}=9.008, p<0.001\right)$ but not conservatism $\left(F_{3,210}=\right.$ 1.082, $p=0.358$ ). Post-hoc comparisons using the Tukey HSD test revealed that the highly delusional group had significantly higher evidence integration scores (mean $=0.581$, s.D. $=1.462,95 \%$ CI $0.296-0.866$ ) than all other groups [(1) low-delusional: mean $=-0.025$, S.D. $=0.808,95 \%$ CI -0.195 to 0.144 , (2) OCD: mean $=-0.493$, s.D. $=0.659,95 \%$ CI -0.911 to -0.075 , and (3) healthy controls: mean $=-0.401$, s.D. $=$ $0.654,95 \%$ CI -0.743 to -0.060$)$; all $\left.p^{\prime} s<0.005\right]$. Comparisons between low-delusional, OCD and healthy control groups were not significant (all $p^{\prime} \mathrm{s}>$ 0.15). These relationships are presented in Fig. 2.

Given the significant group effect observed for verbal IQ (driven by high-delusional subjects having a lower mean IQ than low-delusional subjects), there was a concern that IQ may be contributing to the group differences observed for evidence integration. However, a reanalysis using MANCOVA with IQ entered as a covariate showed that the significant main effect of group remained $\left(\Lambda_{\text {Wilks }}=0.877, F_{6,412}=\right.$ $4.672, p<0.001)$, and a follow-up ANCOVA showed that the between-groups effect for evidence integration remained significant when IQ was entered as a covariate $\left(F_{3,212}=8.706, p<0.001\right)$.

Given the overlap commonly observed between the presence of delusions and hallucinations (Liddle, 1987; Woodward et al. 2003, 2004, 2013, in press; van der Gaag et al. 2006), the specificity of the evidence integration effect to delusions was examined by performing independent $t$ tests between high- and lowhallucinating schizophrenia patients, using the same cut-off as was used to classify the highly delusional schizophrenia group (PANSS score $\geqslant 4$ ). Neither evidence integration nor conservatism differed between high-hallucinating $(n=36 ; \quad$ delusional $=23$, non-delusional=13) and low-hallucinating $(n=127$; delusional $=20$, non-delusional $=107$ ) subgroups for hallucinations (both $p^{\prime} \mathrm{s}>0.09$ ).

\section{Discussion}

Previous analyses using the BADE task have demonstrated that delusional schizophrenia patients show a relative unwillingness to down-rate disconfirmed interpretations of a given scenario, illustrating a defining characteristic of delusions in that they are maintained with conviction despite contradictory information (APA, 2000). The present study aimed to verify these previously identified cognitive processes in a large sample and to compare people with schizophrenia to those with OCD patients on these measures. This was achieved using a component analysis of all interpretation ratings. Two components accounted for approximately $67 \%$ of the variance in the BADE task: evidence integration (plausibility ratings reflecting the degree to which disambiguating information has been integrated) and response conservatism (plausibility ratings indexing an unwillingness to provide high ratings when justified). Only the evidence integration component distinguished between severely delusional schizophrenia patients and non-delusional subjects, with delusional subjects giving relatively high plausibility ratings for disconfirmed interpretations and low ratings for confirmed (true) interpretations. The absence of a difference in evidence integration between high- and low-hallucinating patients suggests that this effect is specific to delusions. Conservatism scores did not differ between groups, arguing against a generalized performance bias. 


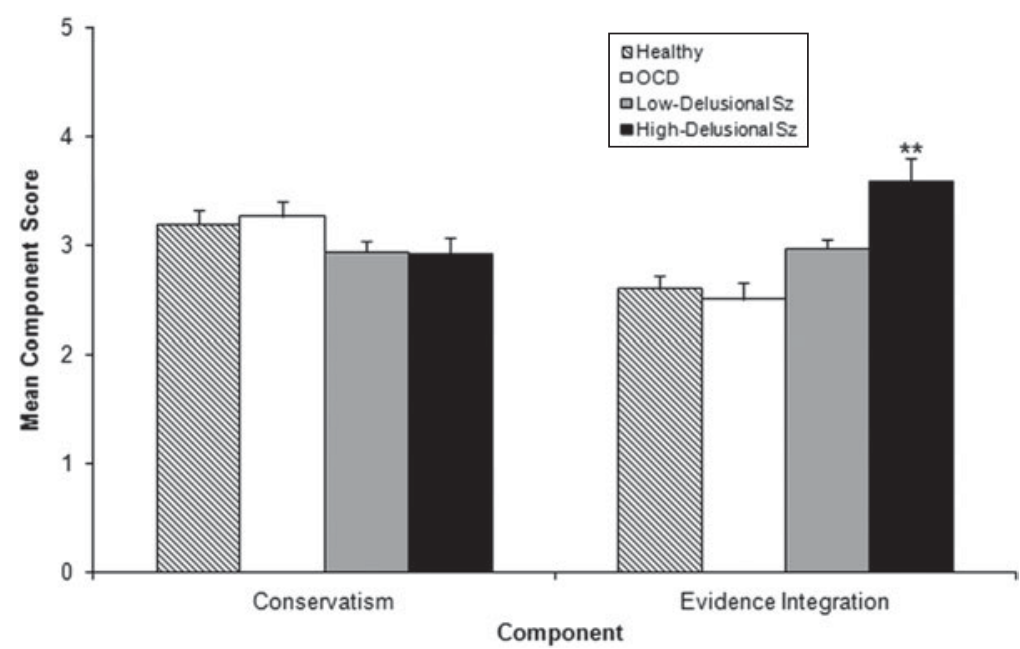

Fig. 2. Mean component scores plotted as a function of group [healthy controls, $n=30$; obsessive-compulsive disorder (OCD), $n=20$; low-delusional, $n=121$; high-delusional, $n=43$ ]. The mean of the component scores was rescaled to 3 for display purposes. ${ }^{* *} p<0.005$ for comparisons between high-delusional subjects and all other groups.

\section{Comparisons with previous studies}

In the previous study from which some of the present data were derived (Veckenstedt et al. 2011), a BADE was measured by lure rating adjustments, and this was not enhanced in delusional patients compared with non-delusional patients. This univariate method of analysis as an index of BADE may sacrifice reliability; for example, the use of raw difference scores is limited because this measure prevents the consideration of participants' initial ratings, and how willing participants are to adjust lure interpretation ratings relative to their adjustments for true/absurd ratings. Moreover, difference scores are less reliable than either of the individual scores from which they are derived because any common variance in the two scores cancels out, and as a result, the error variance constitutes a larger proportion of the total variance. By contrast, aggregates of variables (e.g. component scores, which are variable aggregates weighted by coefficients derived from PCA) are more reliable than any of the individual scores because error variance in the two scores cancels out, and the common variance constitutes a larger proportion of the total variance (Anastasi \& Urbina, 1997). The current study demonstrates how a multivariate approach can be a more informative evaluation of cognitive processes underlying the BADE task. For comparisons across studies, future studies using the BADE task could compute evidence integration and conservatism scores as sums of their respective indicators. Specifically, we recommend that evidence integration be computed as the sum of absurd 1, absurd 2, absurd 3, neutral lure 3, emotional lure 3 , and (true $3 \times-1$ ), and that conservatism be computed as the sum of neutral lure 1 , neutral lure 2 , emotional lure 1, emotional lure 2, true 1, and true 2.
The results from this study do not point to a specific cognitive mechanism affecting evidence integration, although our previously proposed concepts of liberal acceptance (LA; Moritz \& Woodward, 2004; Moritz et al. 2007, 2008) and/or hypersalience of evidencehypothesis (EVH) matches (Speechley et al. 2010; Balzan et al. 2012, 2013) may underlie impairment in evidence integration on the BADE task. For example, the LA account would suggest that, in highly delusional patients, all BADE interpretations are considered somewhat plausible initially, and so the true interpretation is not fully accepted and the absurd/lure interpretations are not fully dismissed, and a definitive decision is delayed. Another possibility is that EVH matches between the initial evidence and the lure interpretations (i.e. hypotheses) result in delusional patients continuing to find these matches hypersalient after the second and third sentences, resulting in a relative reduction in weighting of new evidence. Future BADE experiments including a manipulation of the initial plausibilities of each interpretation, and/or the strength of evidence, would be useful for testing the involvements of LA and EVH matches.

\section{Limitations}

It is important to note that the present study used a cross-sectional design, which is not optimal for studying associations of cognitive biases with psychotic symptoms. Given that symptoms cycle through active states and remissions in schizophrenia, longitudinal studies investigating correlations between change in delusions and change in BADE measures would be a more rigorous way to investigate whether this is a state-dependent cognitive process that changes with fluctuations in symptom severity, or is a trait 
characteristic of patients prone to severe pathology. This link between changes in cognition and changes in symptom severity has been demonstrated with the jumping-to-conclusions (JTC) bias (Woodward et al. 2009; Sanford et al. 2013), a distinct but related cognitive process thought to be associated with delusions (Garety et al. 1991; Fine et al. 2007; Freeman et al. 2008; Speechley et al. 2010; Menon et al. 2013).

\section{Conclusions}

The components derived from the present analysis agree with those previously reported (Speechley et al. 2012) and provide further support for a deficit in evidence integration that is enhanced in currently delusional patients. Importantly, the present study not only replicates this finding in a larger sample of schizophrenia patients but also suggests that BADE is specific to psychosis; that is, the absence of a deficit in evidence integration in OCD patients, who generally display obsessive beliefs and rigidity in cognition but were not experiencing psychosis-like delusions, provides support for the idea that BADE may underlie psychotic delusions in particular rather than some general tendency to persist in dysfunctional thinking.

\section{Implications}

The development of cognitive measures connecting the clinical symptoms of psychosis to the daily experiences of schizophrenia patients may provide significant advances in mental health; for example, symptombased cognitive measures can be adapted to a clinical setting such as metacognitive training, a treatment that aims to reduce the impact of delusional ideation by helping patients recognize and counter the cognitive processes characterizing their illness without discussing personal delusions (Moritz et al. 2011). Such cognitive-focused interventions are crucial because of the limited effectiveness of pharmacological treatments (Elkis, 2007; Leucht et al. 2009) and low medication compliance in psychosis (Byerly et al. 2007). Thus, the BADE represents a promising area of focus for cognitive-based interventions and assessing treatment responsiveness in psychosis.

\section{Acknowledgements}

This work was supported by a grant from the German Research Foundation (Mo 969/6-1) and a National Alliance for Research on Schizophrenia and Depression (NARSAD) grant from the Brain and Behavior Research Foundation.

\section{Declaration of Interest}

None.

\section{References}

Anastasi A, Urbina S (1997). Psychological Testing. Prentice Hall: Upper Saddle River, NJ.

APA (2000). Diagnostic and Statistical Manual of Mental Disorders, Fourth Edition, Text Revision. DSM-IV-TR. American Psychiatric Association: Washington, DC.

Balzan RP, Delfabbro PH, Galletly C, Woodward TS (2012). Reasoning heuristics across the psychosis continuum: the contribution of hypersalient evidence-hypothesis matches. Cognitive Neuropsychiatry 17, 431-450.

Balzan RP, Delfabbro PH, Galletly CA, Woodward TS (2013). Confirmation biases across the psychosis continuum: the contribution of hypersalient evidence-hypothesis matches. British Journal of Clinical Psychology 52, 53-69.

Bandalos D, Boehm-Kaufman M (2008). Four common misconceptions in exploratory factor analysis. In Statistical and Methodological Myths and Urban Legends: Doctrine, Verity and Fable in the Organizational and Social Sciences (ed. C. E. Lance and R. J. Vandenberg), pp. 61-87. Taylor \& Francis Group: New York, NY.

Barlow DH (2002). Anxiety and Its Disorders: The Nature and Treatment of Anxiety and Panic. Guilford Press: New York.

Barrett SL, Mulholland CC, Cooper SJ, Rushe TM (2009). Patterns of neurocognitive impairment in first-episode bipolar disorder and schizophrenia. British Journal of Psychiatry 195, 67-72.

Buchy L, Woodward TS, Liotti M (2007). A cognitive bias against disconfirmatory evidence (BADE) is associated with schizotypal traits. Schizophrenia Research 90, 334-337.

Byerly MJ, Nakonezny PA, Lescouflair E (2007). Antipsychotic medication adherence in schizophrenia. Psychiatric Clinics of North America 30, 437-452.

Cliff N (1988). The eigenvalues-greater-than-one rule and the reliability of components. Psychological Bulletin 103, 276-279.

Colbert S, Peters ER, Garety PA (2010). Delusions and belief flexibility in psychosis. Psychology and Psychotherapy 83, 45-57.

Elkis H (2007). Treatment-resistant schizophrenia. Psychiatric Clinics of North America 30, 511-533.

Fine C, Gardner M, Craigie J, Gold I (2007). Hopping, skipping or jumping to conclusions? Clarifying the role of the JTC bias in delusions. Cognitive Neuropsychiatry 12, 46-77.

Freeman D, Garety PA, Kuipers E, Fowler D, Bebbington PE (2002). A cognitive model of persecutory delusions. British Journal of Clinical Psychology 41, 331-347.

Freeman D, Pugh K, Garety PA (2008). Jumping to conclusions and paranoid ideation in the general population. Schizophrenia Research 102, 254-260.

Garety PA, Hemsley DR, Wessely S (1991). Reasoning in deluded schizophrenic and paranoid patients: biases in 
performance on a probabilistic inference task. Journal of Nervous and Mental Disease 179, 194-201.

Garety PA, Kuipers E, Fowler D, Freeman D, Bebbington PE (2001). A cognitive model of the positive symptoms of psychosis. Psychological Medicine 31, 189-195.

Hayton JC, Allen DG, Scarpello V (2004). Factor retention decisions in exploratory factor analysis: a tutorial on parallel analysis. Organizational Research Methods 7, 191-205.

Jackson DA (1993). Stopping rules in principal components analysis: a comparison of heuristical and statistical approaches. Ecology 74, 2204-2214.

Kay SR, Opler LA, Lindenmayer JP (1989). The Positive and Negative Syndrome Scale (PANSS): rationale and standardisation. British Journal of Psychiatry 155, 59-67.

Leucht S, Arbter D, Engel RR, Kissling W, Davis JM (2009). How effective are second-generation antipsychotic drugs? A meta-analysis of placebo-controlled trials. Molecular Psychiatry 14, 429-447.

Liddle PF (1987). The symptoms of chronic schizophrenia: a re-examination of the positive-negative dichotomy. British Journal of Psychiatry 151, 145-151.

Loong J (1991). Wisconsin Card Sorting Test-IBM Version. Wang Neuropsychological Laboratories: San Luis Obispo, CA.

Menon M, Quilty LC, Zawadzki JA, Woodward TS, Sokolowski HM, Boon HS, Wong AH (2013). The role of cognitive biases and personality variables in subclinical delusional ideation. Cognitive Neuropsychiatry 18, 208-218.

Moritz S, Veckenstedt R, Randjbar S, Vitzthum F, Woodward TS (2011). Antipsychotic treatment beyond antipsychotics: metacognitive intervention for schizophrenia patients improves delusional symptoms. Psychological Medicine 41, 1823-1832.

Moritz S, Woodward TS (2004). Plausibility judgment in schizophrenic patients: evidence for a liberal acceptance bias. German Journal of Psychiatry 7, 66-74.

Moritz S, Woodward TS (2006a). A generalized bias against disconfirmatory evidence in schizophrenia. Psychiatry Research 142, 157-165.

Moritz S, Woodward TS (2006b). Metacognitive control over false memories: a key determinant of delusional thinking. Current Psychiatry Reports 8, 184-190.

Moritz S, Woodward TS, Jelinek L, Klinge R (2008). Memory and metamemory in schizophrenia: a liberal acceptance account of psychosis. Psychological Medicine 38, 825-832.

Moritz S, Woodward TS, Lambert M (2007). Under what circumstances do patients with schizophrenia jump to conclusions? A liberal acceptance account. British Journal of Clinical Psychology 46, 127-137.

Reitan RM, Wolfson D (1985). The Halstead-Reitan Neuropsychological Test Battery. Neuropsychological Press: Tucson, AZ.

Riccaboni R, Fresi F, Bosia M, Buonocore M, Leiba N, Smeraldi E, Cavallaro R (2012). Patterns of evidence integration in schizophrenia and delusion. Psychiatry Research 200, 108-114.
Sanford N, Lecomte T, Leclerc C, Wykes T, Woodward TS (2013). Change in jumping to conclusions linked to change in delusions in early psychosis. Schizophrenia Research 147, 207-208.

Sheehan DV, Lecrubier Y, Sheehan KH, Amorim P, Janavs J, Weiller E, Hergueta T, Baker R, Dunbar GC (1998). The Mini-International Neuropsychiatric Interview (M.I.N.I.): the development and validation of a structured diagnostic psychiatric interview for DSM-IV and ICD-10. Journal of Clinical Psychiatry 59, 22-33.

So S, Garety PA, Peters ER, Kapur S (2010). Do antipsychotics improve reasoning biases? A review. Psychosomatic Medicine 72, 681-693.

Speechley WJ, Ngan ET-C, Moritz S, Woodward TS (2012). Impaired evidence integration and delusions in schizophrenia. Journal of Experimental Psychopathology 3, 688-701.

Speechley WJ, Whitman JC, Woodward TS (2010). The contribution of hypersalience to the 'jumping to conclusions' bias associated with delusions in schizophrenia. Journal of Psychiatry and Neuroscience 35, 7-17.

van der Gaag M, Hoffman T, Remijsen M, Hijman R, de Haan L, van Meijel B, van Harten PN, Valmaggia $L$, de Hert M, Cuijpers A, Wiersma D (2006). The five-factor model of the Positive and Negative Syndrome Scale II:

a ten-fold cross-validation of a revised model. Schizophrenia Research 85, 280-287.

Veckenstedt R, Randjbar S, Vitzthum F, Hottenrott B, Woodward TS, Moritz S (2011). Incorrigibility, jumping to conclusions, and decision threshold in schizophrenia. Cognitive Neuropsychiatry 16, 174-192.

Woodward TS, Buchy L, Moritz S, Liotti M (2007). A bias against disconfirmatory evidence is associated with delusion proneness in a nonclinical sample. Schizophrenia Bulletin 33, 1023-1028.

Woodward TS, Jung K, Hwang H, Yin J, Taylor L, Menon M, Peters E, Kuipers E, Waters F, Lecomte T, Sommer I, Daalman K, van Lutterveld R, Hubl D, Kindler J, Homan P, Badcock JC, Chhabra S, Cella M, Keedy S, Allen P, Mechelli A, Preti A, Siddi S, Erickson D (in press). Symptom dimensions of the Psychotic Symptom Rating Scales (PSYRATS) in psychosis: a multi-site study. Schizophrenia Bulletin.

Woodward TS, Jung K, Smith G, Hwang H, Barr AM, Procyshyn RM, Flynn S, van der Gaag M, Honer WG (2013). Symptom changes in five dimensions of the Positive and Negative Syndrome Scales in refractory psychosis. European Archives of Psychiatry and Clinical Neuroscience. Published online: 15 October 2013. doi:10.1007/ s00406-13-460-x.

Woodward TS, Moritz S, Cuttler C, Whitman J (2006). The contribution of a cognitive bias against disconfirmatory evidence (BADE) to delusions in schizophrenia. Journal of Clinical and Experimental Neuropsychology 28, 605-617.

Woodward TS, Moritz S, Menon M, Klinge R (2008). Belief inflexibility in schizophrenia. Cognitive Neuropsychiatry 13, 267-277. 
Woodward TS, Munz M, Leclerc C, Lecomte T (2009).

Change in delusions is associated with change in 'jumping to conclusions'. Psychiatry Research 170, 124-127.

Woodward TS, Ruff CC, Thornton AE, Moritz S, Liddle P (2003). Methodological considerations regarding the association of Stroop and verbal fluency performance with the symptoms of schizophrenia. Schizophrenia Research 61, 207-214.

Woodward TS, Thornton AE, Ruff CC, Moritz S, Liddle PF (2004). Material-specific episodic memory associates of the psychomotor poverty syndrome in schizophrenia. Cognitive Neuropsychiatry 9, 213-227. 